\title{
Echocardiographic Assessment of Right Ventricular Function in Post Thrombolysed Acute Inferior Wall Infarction Patients with Angiographic Correlation
}

\author{
Sabapathy Kannappan ${ }^{1}$ \\ ${ }^{1}$ Department of Cardiology, Government Vellore Medical College \& Hospital, Vellore, Tamil Nadu, India
}

\section{ABSTRACT}

\section{BACKGROUND}

A significant number of patients with acute Inferior wall infarction will have RV dysfunction and most of them have occlusion of either right or left circumflex coronary artery. But RV assessment is not done routinely in echocardiographic examination. Patients present with inferior wall myocardial infarction (IWMI) subsequently develop right ventricular myocardial infarction (RVMI) and have poor prognosis. In this study we wanted to evaluate the incidence of RVMI and the role of echocardiography in assessing RV function and its impact in predicting infarct related artery by angiographic analysis in acute IWMI after thrombolysis.

\section{METHODS}

86 patients with acute IWMI after thrombolysis were randomly selected. Echocardiographic examination of RV function was performed within 72 hours after thrombolysis. We applied RV echo parameters like FAC (Fractional Area Change \%), TAPSE (Tricuspid Annular Plane Systolic Excursion), RV S' (RV Systolic Excursion Velocity) and MPI (Myocardial Performance Index) to assess RV function. 48 patients underwent coronary angiogram which included 10 patients with normal $\mathrm{RV}$ function, rest with RV dysfunction.

\section{RESULTS}

Among 86 patients, we compared 64 patients of RV dysfunction with 22 patients having normal function. Echo indices were significantly lower in RV dysfunction group. FAC ( $27 \pm 5.95$ vs $45 \pm 5.4$ ) TAPSE $(8.5 \pm 2.019 .59 \pm 2.8$ ), RV S' ( $5.78 \pm 1.26$ vs $17.2 \pm 3.5)$ and RVMPI ( $0.22 \pm 0.03$ vs $0.57 \pm 0.03)$. Angiographic analysis of 28 of 38 patients with RV dysfunction showed significant proximal lesion in RCA as compared to only 2 among 10 patients with normal RV function.

\section{CONCLUSIONS}

Conventional echo examination will underestimate RV dysfunction hence we applied echo parameters like FAC, TAPSE, RV S' and RVMPI and a significant proportion of thrombolysed acute IWMI patients were found to have RV dysfunction and it was also helpful in the prediction of infarct related artery which would be predominantly a critical proximal RCA occlusion.

\section{KEY WORDS}

RVMI (Right Ventricular Myocardial Infarction), FAC (Fractional Area Change \%), TAPSE (Tricuspid Annular Plane Systolic Excursion), RV S' (RV Systolic Excursion Velocity) ,MPI (Myocardial Performance Index), IRA ( Infarct Related Artery)
Corresponding Author: Dr. Sabapathy Kannappan, No. 7 / 2A, Vallalar Street, Thiru Nagar , Katpadi, Vellore - 06, Tamil Nadu, India.

E-mail: sabacardio1966@gmail.com

DOI: $10.14260 / j e m d s / 2021 / 493$

How to Cite This Article:

Kannappan S. Echocardiographic assessment of right ventricular function in post thrombolysed acute inferior wall infarction patients with angiographic correlation. J Evolution Med Dent Sci 2021;10(31):2406-2411, DOI: 10.14260/jemds/2021/493

Submission 25-03-2021, Peer Review 29-05-2021, Acceptance 05-06-2021, Published 02-08-2021.

Copyright (C) 2021 Sabapathy Kannappan This is an open access article distributed under Creative Commons Attribution License [Attribution 4.0 International (CC $B Y 4.0)]$ 


\section{BACKGROUND}

Inferior myocardial infarctions are generally viewed as having a more favourable prognosis than anterior wall infarctions. A number of recent trials of thrombolytic therapy in acute inferior wall infarction appear to support this view, with mortality rates of $2-9 \%$. But many of these trials have failed to demonstrate reduced mortality after thrombolytic therapy in a subset of patients who have RVMI and / or RV dysfunction with inferior infarction. It is important to note, that this subset of patients who develop complications with an increased mortality will substantially alter an otherwise favourable prognosis. ${ }^{1}$ Patients with inferior myocardial infarction who have RV myocardial involvement appear to have a worse prognosis than those who do not have RV involvement. These patients appear to be at increased risk of severe complications, such as left ventricular failure, cardiogenic shock and serious ventricular arrhythmia, and even death. ${ }^{2}$

ST - segment elevation in the right precordial lead, V4R, is one of the most reliable ECG signs of acute RV infarction. It is well understood that ECG evidence of RV infarction is associated with a poor prognosis. 3,4

Pfisterer and associates concluded that RV dysfunction not only results in the occurrence of cardiac death after MI and in addition contributes to LV impairment. 5

A hypokinetic or akinetic segment of the RV observed by echocardiography also could be used to detect RV dysfunction after RV infarction. However, quantitative assessment of RV function is still challenging due to its complex anatomy and thin wall structure, and therefore is not incorporated into daily clinical practice. Echocardiographic assessment of RV - EF is difficult and underlies several limitations. ., $7^{2}$

RV systolic function has been evaluated using several parameters, namely, RVMPI, TAPSE, 2D RV FAC, 2D RV ejection fraction (EF), three-dimensional (3D) RV EF, Tissue Doppler-derived TAPSE (Tricuspid lateral annular systolic velocity $\left(S^{\prime}\right)$ ), and longitudinal strain and strain rate. Among them, more studies have demonstrated the clinical utility and value of RVMPI, TAPSE, 2DFAC, and $S^{\prime}$ of the tricuspid annulus. Although 3D RV EF seems to be more reliable with fewer reproducibility errors, there are insufficient data demonstrating its clinical value at present.

In patients with acute inferior wall myocardial infarction (IWMI), the infarct related artery could be the right coronary artery (RCA) or left circumflex artery (LCX). Acute occlusion of the RCA proximal to the right ventricular branches may result in RV infarction or dysfunction. This identifies a significant subgroup of patients who are associated with considerable immediate morbidity and mortality.

The diagnosis of acute ST - elevation IWMI depends mainly on specific electrocardiographic (ECG) criteria. Predicting the culprit artery in IWMI has been proposed using multiple ECG algorithms, with adequate sensitivity only in patients with extensive ST - segment deviation. ${ }^{8}$

The purpose of this present study was to find out the incidence of RVMI and echocardiographic assessment of RV function in patients with acute IWMI after thrombolysis. We also tried to correlate RV dysfunction and its significance in predicting infarct related artery.

\section{METHODS}

A cross sectional observational type study was conducted from 1st September 2019 to end of February 2020. A total number of 86 patients with ST elevated acute inferior wall myocardial infarction after thrombolysis in ICCU, GVMCH Vellore, randomly selected irrespective of their age, sex, risk factor and treatment were included in this study. Convenient sampling technique (random) was used for the selection of the participants.

\section{Inclusion Criteria}

Patients with ST elevation in ECG lead II, III and aVF for the first time with stable condition who were all thrombolysed with Streptokinase within $6 \mathrm{hrs}$ of onset of symptoms and having an ejection fraction of more than $45 \%$ were included in this study.

\section{Exclusion Criteria}

Acute AWMI, Acute IWMI who were not thrombolysed, patients with coexisting RHD, CHD, moderate to severe PHT, cardiomyopathies, abnormal LV function, LBBB and arrhythmias and poor ECHO window for the assessment of $\mathrm{RV}$ were excluded from the study. Inferior wall myocardial infarction was defined as chest pain with ST - segment elevation of $>0.1 \mathrm{mV}$ in two or more inferior leads (II, III, $\mathrm{aVF}$ ). The presence of $1 \mathrm{~mm}$ ST - segment elevation in lead V4R on admission ECG with hemodynamic compromise like hypotension, elevated JVP and clear lung fields allowed immediate recognition of RV infarction. Echocardiographic examination of RV function was performed in all the patients in the study group within 72 hours after thrombolysis.

The following echocardiographic parameters were applied -

\section{RVFAC (Right Ventricular Fractional Area Change \%)}

RV end diastolic area minus RV end systolic area / end diastolic area X 100 gives RVFAC. Right ventricular area in diastole and systole was obtained by tracing the RV endocardium in both systole and diastole, from the annulus along free wall to apex and then back to annulus along inter ventricular septum in apical four chamber view.

\section{TAPSE (Tricuspid Annular Plane Velocity)}

In apical 4 - chamber view, M - mode cursor was placed through tricuspid annulus at lateral RV free wall in such a way that the annulus moved along $\mathrm{M}$ - mode cursor. From $\mathrm{M}$ mode tracing the amount of longitudinal motion of annulus at peak systole was measured. Total displacement was measured by leading edge of echoes and expressed in millimetre. 


\section{Pulsed Wave Tissue Doppler Imaging}

Pulsed TDI images were acquired by placing TDI cursor on the right ventricular free wall at the level of tricuspid annulus. Being a Doppler based technique, proper alignment (< 20 degree) with ultrasound beam was considered mandatory.

\section{RV Systolic Excursion Velocity (RV S') by Tissue Doppler}

A major positive velocity $\left(S^{\prime}\right)$ was recorded with the movement of annulus towards apex during systole. The measurement of Tissue Doppler-derived right ventricular systolic excursion velocity (RV $S^{\prime}$ ) is similar in many ways to TAPSE, being simple in evaluating longitudinal function and highly reproducible. It uses a pulsed wave (PW) sample volume at the lateral tricuspid annulus from apical transducer position.

\section{Right Ventricular MPI (Myocardial Performance Index) by Tissue Doppler Imaging}

With the movement of annulus towards base during diastole, two major negative waves were recorded, one during early diastole ( $\left.E^{\prime}\right)$ followed by another one during late diastole $\left(A^{\prime}\right)$. $S^{\prime}$ duration was measured as ejection time (ET), the time between the end of $S^{\prime}$ and the beginning of $E^{\prime}$ as isovolumic relaxation time (IRT), time between end of $A^{\prime}$ and beginning of $\mathrm{S}^{\prime}$ as isovolumic contraction time (ICT). Right ventricular MPI was calculated as (IRT + ICT) / ET.

Out of the total number of 86 patients in this study, a subset of 64 patients with IWMI were taken up for diagnostic coronary angiogram with a time period of 7 to 14 days. Significant stenosis was defined as $70 \%$ or greater coronary lumen stenosis, acute thrombosis, or dissected plaque. Proximal RCA lesion was defined as RCA lesion before the origin of acute marginal branch and proximal LCX lesion defined as LCX lesion before the origin of first obtuse marginal branch.

\section{Statistical Analysis}

Quantitative variables were expressed in Mean, Median, Mode and Standard Deviation. Qualitative variables were expressed in chi square test and $P$ value (test of significance).

\section{RESULTS}

Out of 86 patients with acute IWMI, 21 patients were diagnosed to have RVMI based on ECG criteria. Based on echo 22 patients had normal RV function, rest of 64 patients were with RV dysfunction. We had done coronary angiogram for 48 eligible patients among which 10 patients were having normal RV function, rest of 38 patients were with RV dysfunction.

In Angiographic analysis out of 38 patients with RV dysfunction, 24 patients showed significant proximal lesion in RCA as compared to only 2 among 14 patients with normal RV function.

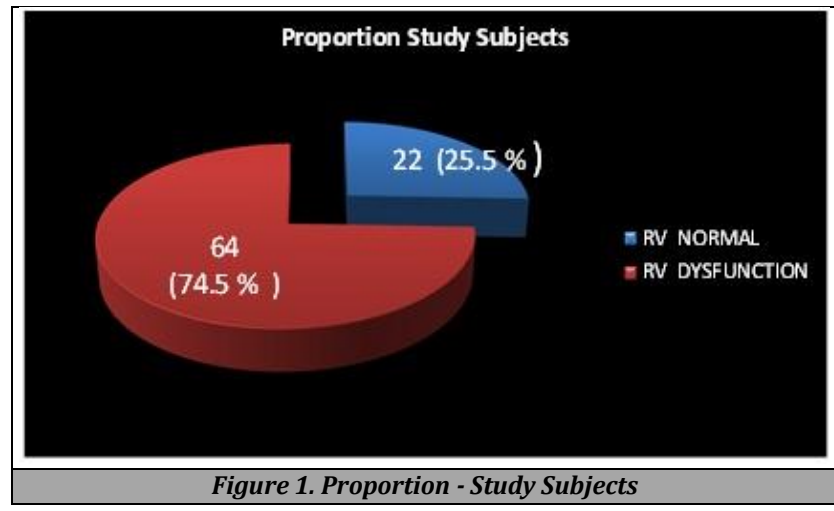

1. RV Normal and RV Dysfunction pattern in this study

(N) 22 - RV normal subjects $25.5 \%$

(N) $64-\mathrm{RV}$ dysfunction subjects

2. FAC among RV dysfunction and RV normal.

$45.3 \%$ - RV normal subjects $(\mathrm{N}=38)$

$27 \%-\mathrm{RV}$ dysfunction subjects $(\mathrm{N}=23)$

In this study RV dysfunction subjects were having lower FAC parameters when compared to RV normal subjects

3. TAPSE ( $\mathrm{mm}$ ) among RV dysfunction and RV normal.

$19.6 \mathrm{~mm}$ - RV normal subjects

$8 \mathrm{~mm}-\mathrm{RV}$ dysfunction subjects

In this study RV dysfunction subjects were having lower TAPSE values when compared to RV normal subjects.

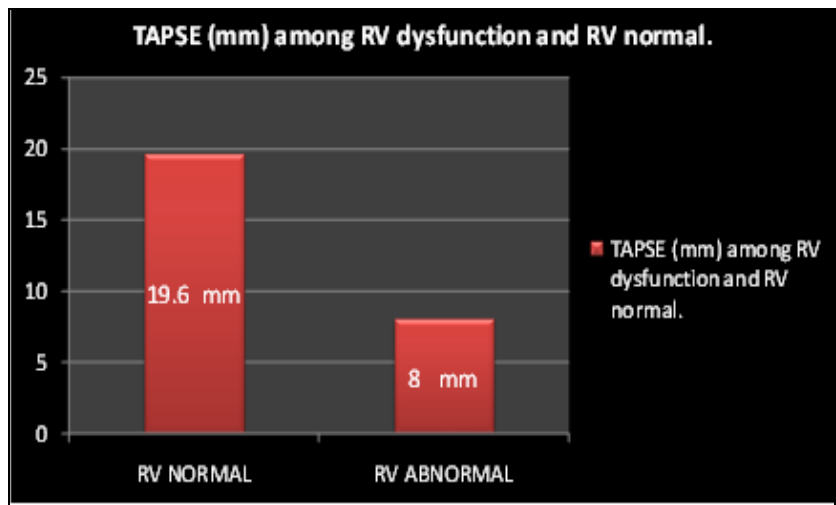

Figure 2. TAPSE (mm) among RV Dysfunction and RV Normal

\begin{tabular}{|ccc|}
\hline & RV Normal & RV Dysfunction \\
\hline Mean & 19.5 & 8.5 \\
Median & 19.5 & 8 \\
Standard Deviation & \pm 2.8 & \pm 2 \\
\hline Table 1. Tapse in Patients with Normal RV Function \\
and RV Dysfunction
\end{tabular}

From the above table the Mean, Median and SD of the above two cardiac parameters are expressed and compared.

4. $\mathrm{RV} \mathrm{S} \mathrm{cm} \mathrm{/} \mathrm{sec} \mathrm{(TDS)}$

$17.8 \mathrm{~cm} / \mathrm{sec}$ - RV normal subjects

$5.8 \mathrm{~cm} / \mathrm{sec}-\mathrm{RV}$ dysfunction subjects

In this study RV dysfunction subjects were having lower RV S values.

\begin{tabular}{|ccc|}
\hline & RV Normal & RV Dysfunction \\
\hline Mean & 17.8 & 5.8 \\
Median & 17.5 & 6 \\
Standard Deviation & \pm 3.3 & \pm 1.25 \\
\hline Table 2. $\mathbf{R V}$ in Patients with Normal RV Function and $\mathbf{R V}$ Dysfunction \\
\hline
\end{tabular}

From the above table the Mean, Median and SD of the above two cardiac parameters are expressed and compared. 
5. RV MPI values among normal and dysfunction RV subjects 0.57 - RV normal subjects

0.20 - RV dysfunction subjects

In this study RV dysfunction subjects were having lower MPI values than RV normal subjects

\begin{tabular}{|ccc|}
\hline & RV Normal & RV Dysfunction \\
Mean & .22 & .57 \\
Median & .20 & .58 \\
Standard Deviation & \pm 3.3 & \pm .03 \\
\hline Table 3. RV MPI Values in Patients with Normal RV Function \\
\multicolumn{3}{c|}{ and RV Dysfunction } \\
\hline
\end{tabular}

From the above table the Mean, Median and SD of the above two scenarios are expressed. The following four parameters (FAC, TAPSE, RV, MPI) on running chi-square test RV dysfunction values were statistically significant with $P$ value $\leq .05$ thereby we are rejecting null Hypothesis and supporting alternate hypothesis.

\section{DISCUSSION}

Inferior Myocardial Infarction (IMI) is estimated to be $40-50$ $\%$ of all acute Myocardial Infarctions (AMI). It is assessed that $30 \%$ of all acute inferior myocardial infarctions of the left ventricle are associated with right ventricular infarction (RVI). The incidence of RVMI is variable depending on the criteria used for detection. Autopsy studies suggest that right ventricular infarction accompanies fatal inferior left ventricular infarction in 24 to 34 percent of cases. ${ }^{9}$

Non invasive studies suggest that RVI occurs in more than 30 percent of patients with acute inferior - posterior left ventricular MI. Anatomic evidence of RVMI is more common than expected hemodynamic pattern. In our study we had applied ECG and hemodynamic criteria to diagnose RVMI in acute IWMI before thrombolysis and it was around around 32 $\%$ which is similar to the study done by Zymer et al.10,11

Dell'Italia el. using right ventricular akinesis or dyskinesis as a marker, found that echocardiography was a highly sensitive technique for detecting hemodynamically significant RVMI. However, it also detected wall motion abnormalities in patients who never developed hemodynamic evidence of RVMI.

This may be due to a superior sensitivity of echocardiography in detecting right ventricular ischemia or infarction that may or may not be associated with hemodynamic derangement. ${ }^{11}$ Lopez - Sendon et al. also obtained similar results in his study. ${ }^{12}$

Inferior acute myocardial infarction (IMI) patients with right ventricular involvement (RVI) have worse outcomes, and furthermore, a significantly increased risk of major complications and in-hospital death have been reported in those patients without reperfusion therapy. ${ }^{13,14,15}$

The regional myocardial function can be quantified with the help of tissue Doppler imaging (TDI), which is a novel ultrasound technique by measuring myocardial velocities. There are 3 available TDI modes: Pulsed wave mode, 2D color mode and color $\mathrm{M}$ - mode by each of which the specific analysis of radial and longitudinal myocardial motion is allowed. 16

The TDI can also be used in the analysis of both phase and amplitude of regional myocardial motion. This is the reason why TDI is considered to be the precise non-invasive method for assessing myocardial dysfunction. ${ }^{17}$

Early identification of RVMI thus has an important role in determining the prognosis and selecting an appropriate treatment strategy in patients with IWMI. ST - segment elevation of $\geq 1.0 \mathrm{~mm}$ in the right precordial chest lead V4R (ST $\uparrow 4 \mathrm{R}$ ) is a simple, readily available, and widely used criterion for RVI.18,19

The echocardiographic indices were used to assess RV function in the study and RVFAC was significantly lower in two third of the patients.

RVFAC has been found to have a good correlation with MRI derived RVEF and is also found to have prognostic significance in patients with myocardial infarction. ${ }^{20}$

Measurement of RVFAC can be difficult when the echo window is poor and correlation between observers is also poor when compared with other indices. ${ }^{21}$ From our study we infer that inpatients with good echo window, RVFAC can be a useful measure to predict RV dysfunction.

TAPSE < $16 \mathrm{~mm}$ indicates RV systolic dysfunction according to ASE guidelines. Earlier studies had shown good correlation of TAPSE with ECG evidence of RV infarction, but the number of patients was less and there was no angiographic correlation. TAPSE was also an independent predictor of mortality in inferior wall MI.22

TAPSE has some limitations in which measurement is restricted to longitudinal function of $\mathrm{RV}$ free wall and functional status of LV may have an influence on it. TAPSE was also found to have a good correlation with radionuclide derived EF. ${ }^{23}$ In our study TAPSE was significantly lower in patients with RV dysfunction.

ASE guidelines suggest a Tissue Doppler systolic velocity namely velocities from RV free wall at the level of tricuspid annulus (RV S') $10 \mathrm{~cm} / \mathrm{s}$ and below showed good correlation with RV dysfunction. ${ }^{24}$

Tissue Doppler systolic annular velocity has also been shown to correlate with prognosis in IWMI.26 Mezulin et al. found out that systolic annular velocity by tissue Doppler correlated with RVEF in that velocity $<11.5 \mathrm{~cm} / \mathrm{s}$ will predict RVEF less than $45 \% .^{25}$

Myocardial performance index by pulsed wave Doppler (MPI - PW) was also found to correlate with RV dysfunction but it was neither correlated with the presence of RVMI nor with angiography in the earlier studies. ${ }^{26}$

MPI calculated in this method was less reliable as it utilized two different cardiac cycles for measurement of time intervals and inter observer correlation was also less compared to other measures. Hence we have taken Tissue Doppler based MPI in our study.

According to ASE guidelines MPI - TDI $>0.55$ is indicative of RV systolic dysfunction. Our findings in the study are concordant with the earlier studies and provide optimal cut off values to predict RV dysfunction.

RVMI generally results from proximal occlusion of the right coronary artery (RCA). ST - segment changes in lead V4R have also emerged as reliable markers of the site of coronary artery occlusion in patients with IWMI; ST $\uparrow$ V4R is useful for identifying proximal occlusion of the RCA, whereas ST - segment depression of $\geq 1.0 \mathrm{~mm}$ in lead V4R suggests occlusion of the left circumflex artery. ${ }^{3,4}$

The left ventricular posterior wall is situated diagonally opposite to the right ventricular anterior wall. ST - segment 
elevation due to transmural ischemia in the posterior wall can therefore reciprocally depress the right precordial ST segment.

The RCA sometimes supplies not only the inferior wall, but also the posterior wall. In patients with such a dominant RCA, proximal occlusion of the RCA can lead to ST - segment changes in the right precordial lead, caused by competition between ST - segment elevation due to RVMI and reciprocal changes due to posterior wall involvement (PWMI), which can result in attenuated right precordial ST - segment elevation. ${ }^{27}$

There is little clinical evidence about the impact of thrombolysis on patients with RV involvement during inferior infarction. Schuler et al. retrospectively studied patients with acute inferior infarction due to proximal right coronary artery occlusion with acute inferior infarction that revealed marked improvement in RV function only in patients with sucessful thrombolysis whereas there was no improvement in RV function in the patients with failed thrombolysis. ${ }^{28}$

These findings are in contrast to those of Verani et al. who found that RV function improved even in those patients without successful thrombolysis. ${ }^{29}$ However, both of these studies were from a small sample size. In our study 22 patients out of 86 had normal RV function and we assume that this could be due to successful lysis.

Data from the TIMI II trial support the view that successful thrombolysis reduces the incidence of RV involvement during inferior infarction. Angiographic analysis of those patients undergoing protocol catheterization after thrombolysis reveals that patients with patency of the infarct related artery had a much lower incidence of RV infarction. In addition, patients with RV infarction were significantly more likely to have occlusion of the infarct-related artery despite thrombolytic therapy than patients without RV involvement. 30

Bowes et al. in his study found that $71 \%$ of the patients with RV dysfunction showed proximal RCA as IRA. It has been found that proximal RCA occlusion typically compromises RV branch perfusion in $56.7 \%$ of cases, resulting in RV ischemic dysfunction, whereas distal RCA occlusions rarely do that. ${ }^{31}$ In our study out of 38 patients with RV dysfunction, 24 patients $(64 \%)$ had significant proximal lesions in the infarct related artery mainly RCA when compared to only 2 out of 10 (20\%) patients with normal RV function. This is in concordance with study done by Rajesh et al. ${ }^{32}$

\section{CONCLUSIONS}

The presence of RV dysfunction in acute IWMI will lead to increased risk of shock, arrhythmia and death. Thus, the need for diagnosis of RV dysfunction is evident but conventional echo examination will underestimate RV dysfunction. In this study, we applied echo parameters like FAC, TAPSE, RV S' and RVMPI to assess RV function. We found that significant proportion of thrombolysed acute IWMI patients were found to have RV dysfunction and were helpful in the prediction of infarct related artery which would predominantly be a critical proximal RCA occlusion. This stable high-risk subgroup of patients with acute inferior wall myocardial infarction should be considered as high - priority candidates for reperfusion.

\section{Limitations}

A small number of patients is one of the limitations of this study. We were not able to include the influence of drugs that were used along with thrombolysis is another limitation.

Data sharing statement provided by the authors is available with the full text of this article at jemds.com.

Financial or other competing interests: None.

Disclosure forms provided by the authors are available with the full text of this article at jemds.com.

\section{REFERENCES}

[1] Berger PB, Ryan TJ. Inferior myocardial infarction. Highrisk subgroups. Circulation 1990;81(2):401-11.

[2] Bueno H, Lopez-Palop R, Bermejo J, et al. In-hospital outcome of elderly patients with acute inferior myocardial infarction and right ventricular involvement. Circulation 1997;96(2):436-41.

[3] Braat SH, Brugada P, De Zwaan C, et al. Value of electrocardiogram in detecting right ventricular involvement in patients with acute inferior wall myocardial infarction. Heart J 1983;49(4):368-77.

[4] Zehender M, Kasper W, Kauder E, et al. Right ventricular infarction as an independent predictor of prognosis after acute inferior myocardial infarction. $\mathrm{N}$ Engl J Med 1993;328(14):981-8.

[5] Pfisterer M, Emmenegger H, Müller-Brand J, et al. Prevalence and extent of right ventricular dysfunction after myocardial infarction-relation to location and extent of infarction and left ventricular function. Int J Cardiol 1990;28(3):325-32.

[6] Lopez-Sendon J, Garcia-Fernandez MA, Coma-Canella I, et al. Segmental right ventricular function after acute myocardial infarction: two-dimensional echocardiographic study in 63 patients. Am J Cardiol 1983;51(3):390-6.

[7] Dambrauskaite V, Delcroix M, Claus P, et al. Regional right ventricular dysfunction in chronic pulmonary hypertension. J Am Soc Echocardiogr 2007;20(10):117280.

[8] El Sebaie MH, El khateeb O. Right ventricular echocardiographic parameters for prediction of proximal right coronary artery lesion in patients with inferior wall myocardial infarction. J Saudi Heart Assoc 2016;28(2):73-80.

[9] Andersen HR, Falk E, Nielsen D. Right ventricular infarction: frequency, size and topography in coronary heart disease: a prospective study comprising 107 consecutive autopsies from a coronary unit. J Am Coll Cardiol 1987;10(6):1223-32.

[10] Zeymer U, Neuhaus KL, Wegscheider K, et al. Effects of thrombolytic therapy in acute inferior myocardial infarction with or without right ventricular involvement. HIT-4 trial group. Hirudin for improvement of thrombolysis. J Am Coll Cardiol 1998;32(4):876-81. 
[11] Dell'Italia LJ, Starling MR, Crawford MH, et al. Right ventricular infarction: identification by hemodynamic measurements before and after volume loading and correlation with noninvasive techniques. J Am Coll Cardiol 1984;4(5):931-9.

[12] Lopez-Sendon J, Garcia-Fernandez MA, Coma-Canella I, et al. Segmental right ventricular function after acute myocardial infarction: two-dimensional echocardiographic study in 63 patients. J Am Coll Cardiol1983;51:390-6.

[13] Ninkovic VM, Perunicic JZ, Ninkovic SM, et al. Predictors of in-hospital mortality in patients with acute inferior infarction of the left ventricle accompanied by right ventricular infarction when treated with percutaneous coronary intervention. J Clin Exp Cardiol 2013;4:7.

[14] Zehender M, Kasper W, Kauder E, et al. Eligibility for and benefit of thrombolytic therapy in inferior myocardial infarction: focus on the prognostic importance of right ventricular infarction. J Am Coll Cardiol 1994;24(2):3629.

[15] Zornoff LA, Skali H, Pfeffer MA, et al. Right ventricular dysfunction and risk of heart failure and mortality after myocardial infarction. J Am Coll Cardiol 2002;39(9):1450-5.

[16] Bleeker GB, Steendijk P, Holman ER, C et al. Assessing right ventricular function: the role of echocardiography and complementary technologies. Heart 2006;92(Suppl 1):119-26.

[17] Chetboul V. Tissue doppler imaging: a promising technique for quantifying regional myocardial function. J Vet Cardiol 2002;4(2):7-12.

[18] Wellens HJ. The value of the right precordial leads of the electrocardiogram. N Engl J Med 1999;340(5):381-3.

[19] Klein HO, Tordjman T, Ninio R, et al. The early recognition of right ventricular infarction: diagnostic accuracy of the electrocardiographic V4R lead. Circulation 1983;67(3):558-65.

[20] Anavekar NS, Gerson D, Skali H, et al. Two-dimensional assessment of right ventricular function: an echocardiographic-MRI correlative study. Echocardiography 2007;24(5):452-6.

[21] Anavekar NS, Skali H, Bourgoun M, et al. Usefulness of right ventricular fractional area change to predict death, heart failure and stroke following myocardial infarction (from the VALIANT ECHO Study). Am J Cardiol 2008;101(5):607-12.

[22] Alam M, Wardell J, Andersson E, et al. Right ventricular function in patients with first inferior myocardial infarction: assessment by tricuspid annular motion and tricuspid annular velocity. Am Heart J 2000;139(4):7105.

[23] Kaul S, Tei C, Hopkins JM, et al. Assessment of right ventricular function using two-dimensional echocardiography. Am Heart J 1984;107(3):526-31.

[24] Dokainish H, Abbey H, Gin K, et al. Usefulness of tissue doppler imaging in the diagnosis and prognosis of acute right ventricular infarction with inferior wall acute left ventricular infarction. Am J Cardiol 2005;95(9):1039-42.

[25] Meluzin J, Spinarova L, Bakala J, et al. Pulsed doppler tissue imaging of the velocity of tricuspid annular systolic motion. a new, rapid and non-invasive method of evaluating right ventricular systolic function. Eur Heart J 2001;22(1):340-44.

[26] Chockalingam A, Gnanavelu G, Alagesan R, et al. Myocardial performance index in evaluation of acute right ventricular myocardial infarction. Echocardiography 2004;21(6):487-94.

[27] Kosuge M, Kimura K, Ishikawa T, et al. Implications of the absence of ST-segment elevation in lead V4R in patients who have inferior wall acute myocardial infarction with right ventricular involvement. Clin Cardiol 2001;24(3):225-30.

[28] Schuler G, Hoffman M, Schwarz F, et al. Effect of successful thrombolytic therapy on right ventricular function in acute inferior wall myocardial infarction. Am J Cardiol 1984;54(8):951-7.

[29] Verani MS, Tortoledo FE, Batty JW, et al. Effect of coronary artery recanalization on right ventricular function in patients with acute myocardial infarction. JAm Coll Cardiol 1985;5(5):1029-35.

[30] Berger PB, Ruocco NA Jr, Ryan T], et al. Frequency and significance of right ventricular dysfunction during inferior wall left ventricular myocardial infarction treated with thrombolytic therapy (results from the Thrombolysis In Myocardial Infarction [TIMI] II trial). Am J Cardiol 1993;71(13):1148-52.

[31] Bowers TR, O'Neill WW, Pica M, et al. Patterns of coronary compromise resulting in acute right ventricular ischemic dysfunction. Circulation 2002;106(9):1104-9.

[32] Rajesh GN, Raju D, Nandan D, et al. Echocardiographic assessment of right ventricular function in inferior wall myocardial infarction and angiographic correlation to proximal right coronary artery stenosis. Indian Heart J 2013;65(5):522-8. 